\title{
A Low Cost Alternative Method for Harmonics Estimation in a BIST context
}

\author{
V. Fresnaud ${ }^{1,2}$, L. Bossuet ${ }^{2}$, D. Dallet ${ }^{2}$, S. Bernard ${ }^{3}$, J.M. Janik ${ }^{4}$, B. Agnus ${ }^{1}$, Ph. Cauvet $^{1}$, Ph. Gandy ${ }^{1}$ \\ ${ }^{1}$ Philips France Semiconducteurs, 2 Rue de la Girafe B.P. 512014079 Caen Cedex 5, France \\ ${ }^{2}$ Laboratoire IXL - ENSEIRB - CNRS UMR 5818, 351 cours de la Libération, 33405 Talence, France \\ ${ }^{3}$ LIRMM, University of Montpellier, CNRS - 161 rue Ada, 34392 Montpellier, France \\ ${ }^{4}$ GREYC,University of Caen, CNRS - 6 bd Maréchal Juin, 14050 Caen, France \\ vincent.fresnaud@philips.com
}

\begin{abstract}
Spectral analysis represents a key component in signal processing. The on-chip implementation of classical spectral estimation techniques is generally not considered as a viable BIST solution because of the huge amount of required additional circuitry (multipliers, complex operators). This paper describes a new method for spectral parameter estimation allowing the extraction of the first harmonic with only very simple operators. This method is based on Fourier series expansion and a piecewise algorithm. The results are validated using simulations and experiments, and the method showed relevant results to an embedded solution.
\end{abstract}

\section{Introduction}

With the advances on analogue-digital ICs, faster and more complex test equipment is required to meet ever more severe test specifications. An attractive alternative to simplify the test equipment is to move some or all the tester functions onto the chip itself. The use of Built In Self Test (BIST) for high volume production of mixed signal ICs is desirable to reduce the cost per chip during production testing by the manufacturers.

Spectral analysis represents a key component in signal processing. The power spectrum contains a large set of information that can be used, in electronic devices characterisation. For instance, spectral measurements like SNR, THD allow test engineers to sort out analogue or mixedsignal integrated circuits in production test. In recent papers $[1,2]$, spectral analysis has been used to estimate the whole set of ADC (Analogue-to-Digital Converter) performances. Moreover, as in the case of ADC testing, power estimation of the first harmonics is generally sufficient.

In this context, our objective is to develop a technique for harmonic estimation requiring minimal hardware resources in order to propose a viable BIST implementation.
Actually, spectral analysis has led to many different power spectrum estimation methods, which could be divided in two parts: parametric and non-parametric $[3,4,5]$.

Parametric methods consist in modelling the data by a small set of parameters. The autoregressive (AR) model is the most commonly used model. Finding the parameters leads to complex algorithm [13], which usually required matrix inversion and/or single value decomposition algorithms. Those methods give very accurate results in terms of frequency estimation but are very time consuming and due to their complex processing, could not be used for an embedded process. In this class of parametric techniques, some embedded solutions were proposed to estimate directly the signal harmonics. In [6], authors propose a digital filter bank tuned on the frequency of the harmonics to be evaluated. This technique could be implanted with minimum silicon area overhead if only the first main (1 to 5) harmonics are considered. But the harmonic estimation accuracy is too sensitive to variation of the input signal frequency. It is possible to add some adaptive circuitries to make this technique more robust [7], but this solution involves a lot of hardware resources unsuitable for BIST context. Another solution to evaluate harmonics consists in using a simple $\Sigma-\Delta$ modulator $[8,9]$. This technique is really attractive but it is only usable on an analog signal and not on the digital output of ADC.

Non-parametric methods are based on Fourier analysis, which is usually implemented by means of Fast Fourier Transform (FFT) algorithm [12]. The FFT algorithm gives an accurate estimation of the spectrum, but the huge amount of multipliers required for an embedded process is not viable in a BIST context. For instance a Radix-4 FFT algorithm applied to an acquisition length of 4096 real samples, need more than 50, 000 products and 120, 000 adds [14]. In this paper, we propose a new power estimation algorithm of the first harmonics that respects the previously enounced constraints. The method is based on discrete Fourier series expansion model, and use piecewise signal decomposition 
and a very simple processing. The paper is organized as follows. Section 2 presents the technique we propose. Section 3 gives simulation results and comparisons with the most common algorithm: FFT. Finally, section 4 concludes this paper.

\section{Method for harmonic estimation}

\subsection{Discrete Fourier series expansion}

The method is based on the description of the analysed signal by a discrete Fourier series expansion. Any Tperiodic signal $s(t)$ could be expressed as a Fourier series:

$$
s(t)=\frac{a_{0}}{2}+\sum_{k=1}^{\infty}\left(a_{k} \cos (k \omega t)+b_{k} \sin (k \omega t)\right)
$$

with $\omega=\frac{2 \pi}{T}$ and $a_{k}, b_{k}$ are the Fourier series coefficients defined by:

$$
\begin{aligned}
a_{k} & =\frac{1}{T_{s}} \cdot \int_{0}^{T_{s}} s(t) \cdot \cos (k \omega t) d t \\
b_{k} & =\frac{1}{T_{s}} \cdot \int_{0}^{T_{s}} s(t) \cdot \sin (k \omega t) d t
\end{aligned}
$$

In the context of digital processing, discrete signal has to be considered. When digitising a signal, coherent sampling is needed to eliminate the used of a time windowing by guaranteeing that the sample set contains a complete periodic waveform representation [15]. Coherent sampling defines a relationship between the sampling frequency $F_{s}$ (or the sampling period $T_{s}$ ), the number of samples Ns, the test signal frequency $F_{t}$ (or the corresponding period $T$ ) and the number of test signal periods sampled $M$. The coherency formula is given by:

$$
M . F_{s}=N_{s} \cdot F_{t} \quad \text { or } \quad M . T=N_{s} \cdot T_{s}
$$

where $M$ and $N$ are mutually prime to assure unique sample points.

With these new conditions we can define the Unit Test Period $(U T P)$ [11], which represents the time required to take all samples, and its associated frequency, $F_{r e s}$, the frequency resolution:

$$
U T P=\frac{M}{F_{t}}=\frac{N}{F_{s}}, F_{r e s}=\frac{1}{U T P}=\frac{F_{t}}{M}=\frac{F_{s}}{N}
$$

A FFT operation on a set of coherent samples puts all relevant information about the fundamental and harmonics of the signal into frequency ranges of equal width. These frequency ranges are called bins. The width of the bins is determined by the frequency resolution Fres. Due to Nyquist, the total number of frequency bins used for spectral analysis is $N s / 2$, and as their width is $F_{r e s}$, we can state that the maximum frequency in the spectral data is :

$$
F_{t_{M A X}}=F_{r e s} \cdot \frac{N_{s}}{2}
$$

So, for a discrete signal that respects the previous conditions, the formula of the Fourier series becomes:

$$
\begin{array}{r}
s[n] \approx \frac{a_{0}}{2}+\sum_{k=1}^{\frac{N_{s}}{2}}\left(a_{k} \cos (k \omega n)+b_{k} \sin (k \omega n)\right) \\
n \in\left[1 . . N_{s}\right]
\end{array}
$$

with

$$
\omega=\frac{2 \pi}{U T P}=\frac{2 \pi}{N_{s} T_{s}}
$$

Generally the most part of the signal power is concentrated in the first harmonics. Consequently, we can estimate the signal in considering the first $K_{M A X}$ harmonics with following expression:

$$
\begin{array}{r}
s[n] \approx \frac{a_{0}}{2}+\sum_{k=1}^{K_{M A X}}\left(a_{k} \cos (k \omega n)+b_{k} \sin (k \omega n)\right) \\
n \in\left[1 . . N_{s}\right]
\end{array}
$$

$a_{k}$ and $b_{k}$ are then evaluated using classical expressions

$$
\begin{array}{r}
a_{k}=\frac{1}{N_{s} \cdot T_{s}} \cdot \sum_{j=1}^{N_{s}} s[n] \cdot \cos \left(2 \pi \frac{k}{N_{s} \cdot T_{s}} n\right) \\
k \in\left\{1, \ldots, K_{M A X}\right\} \\
b_{k}=\frac{1}{N_{s} \cdot T_{s}} \cdot \sum_{j=1}^{N_{s}} s[n] \cdot \sin \left(2 \pi \frac{k}{N_{s} \cdot T_{s}} n\right) \\
k \in\left\{1, \ldots, K_{\text {MAX }}\right\}
\end{array}
$$

The computation of these expressions requires a large number of multiplications. Our main goal is to estimate the values of the parameters $a_{k}$ and $b_{k}$, with original technique requiring minimal hardware resources.

\subsection{Method principle}

Our objective is to minimize the number of complex computations required to estimate the coefficients $a_{k}$ and $b_{k}$. The idea of our method consists in defining very simple computations to extract independently each coefficient $a_{k}$ from a composite signal. We consider that the average value of the analysed signal is null $\left(a_{0}=0\right)$.

The first step consists in distinguishing between the calculation of the coefficients $a_{k}$ and $b_{k}$ in using additional functions $h$ and $g$ defined as follows: 


$$
\begin{aligned}
& g(t)=s(t)+s(-t) \approx 2 \sum_{k=1}^{K_{\text {MAX }}}\left(a_{k} \cos (k \omega t)\right) \\
& h(t)=s(t)-s(-t) \approx 2 \sum_{k=1}^{K_{M A X}}\left(b_{k} \sin (k \omega t)\right)
\end{aligned}
$$

Those functions are easy to compute considering the fact that the function $s$ is a periodic one. In discrete time it comes by symmetry around $N_{s} / 2$ :

$$
\begin{gathered}
g[n]=s\left[\frac{N_{s}}{2}-n+1\right]+s[n] \\
h[n]=s\left[\frac{N_{s}}{2}-n+1\right]-s[n] \text { with } n \in\left[1 . . \frac{N_{s}}{2}\right]
\end{gathered}
$$

To illustrate our method, we focus on the $a_{k}$ estimation method. The estimation of $b_{k}$ coefficients is based on a very similar technique.

The fundamental idea of our method is to reorganize the sample obtained from the signal in order to extract Fourier coefficients with only cumulative sum on samples.

Before describing our method in the general case, let us consider the simple example of the first three harmonic determinations.

2.2.1. Illustration for a third order model. In the case where $K_{M A X}$ is equal to 3 , the signal $g[n]$ is composed of 3 cosines of amplitude ak with $k \in\{1,2,3\}$ as illustrated figure 1. Our goal is to separate each contribution of the cosines in order to estimate their amplitudes $a_{k}$. Let us choose the frequency corresponding to the second wave, i.e. the wave described by $\cos (2 \omega t)$. In order to evaluate the amplitude $a_{2}$, our method consists in multiplying the signal by the sign of $\cos (2 \omega t)$. Figure 2 represents the resultant signal and the influence on the sign of $\cos (2 \omega t)$ on the different cosine functions.

From this figure, we can remark that the average values of $a_{1} \cos (\omega t) \operatorname{sign}[\cos (2 \omega t)]$ and $a 3 \cos (3 \omega t) \operatorname{sign}[\cos (2 \omega t)]$ seem equal to zero. This can be proved thanks to the following equation:

$$
\begin{aligned}
G_{2} & =\int_{0}^{N_{s} T_{s}} g(t) \operatorname{sign}(\cos (2 \omega t)) d t \\
& =\int_{0}^{N_{s} T_{s}} 2 \sum_{k=1}^{3}\left(a_{k} \cos (k \omega t)\right) \operatorname{sign}(\cos (2 \omega t)) d t \\
& =\sum_{k=1}^{3}\left(2 \cdot a_{k} \int_{0}^{N_{s} T_{s}} \cos (k \omega t) \operatorname{sign}(\cos (2 \omega t)) d t\right) \\
& =2 . a_{1} \cdot 0+2 \cdot a_{2} \cdot \frac{2}{\omega}+2 . a_{3} \cdot 0=a_{2} \cdot \frac{4}{\omega}
\end{aligned}
$$

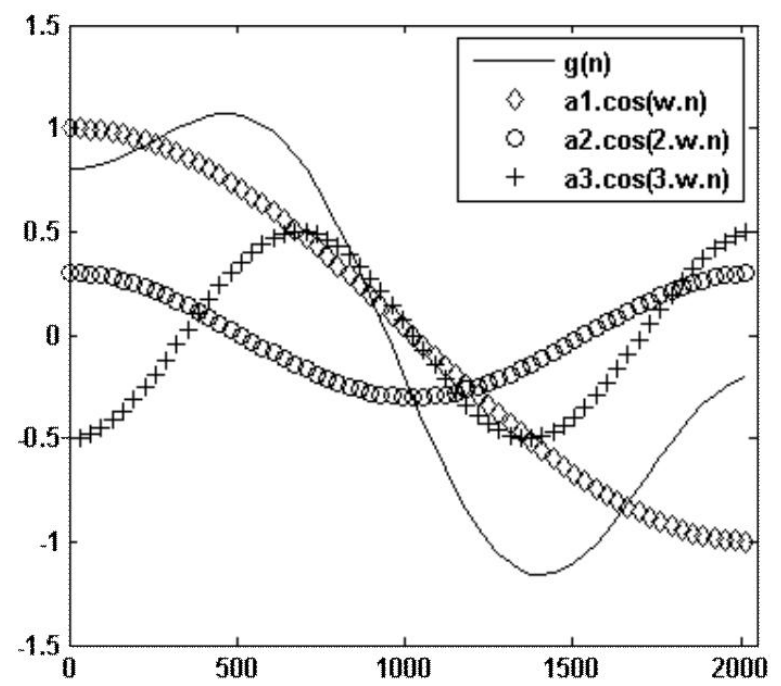

\section{Figure 1. Three cosine signals and associ- ated composite signal $\mathrm{g}[\mathbf{n}]$}

For this specific example, the contribution of $\cos (\omega t)$ and $\cos (3 \omega t)$ in the computation of the parameter $a_{2}$ are nulls. Consequently, if the signal is only made of 3 cosines, multiplying this signal by the sign of $\cos (2 \omega t)$ could allow us to determine the value of the coefficient $a_{2}$ corresponding of the second harmonic amplitude with

$$
a_{2}=\frac{\omega}{4} \cdot \int_{0}^{N_{s} T_{s}} g(t) \cdot \operatorname{sign}(\cos (2 \omega t)) d t
$$

that can be expressed by the following computation for the discrete case:

$$
a_{2}=\frac{\omega}{4}\left(\sum_{0}^{\frac{N_{s}}{8}} g[n]-\sum_{\frac{N_{s}}{8}}^{\frac{3 N_{s}}{8}} g[n]+\sum_{\frac{3 N_{s}}{8}}^{\frac{N_{s}}{2}} g[n]\right)
$$

Based on the same principle, we can extract the amplitude of the third harmonic component, multiplying the signal by the sign of $\cos (3 \omega t)$ :

$$
a_{3}=\frac{\omega}{4}\left(\sum_{0}^{\frac{N_{s}}{12}} g[n]-\sum_{\frac{N_{s}}{12}}^{\frac{3 N_{s}}{12}} g[n]+\sum_{\frac{3 \cdot N_{s}}{12}}^{\frac{5 N_{s}}{12}} g[n]-\sum_{\frac{5 N_{s}}{12}}^{\frac{N_{s}}{2}} g[n]\right)
$$

In the case of $a_{1}$ computation the result is not so trivial. The third harmonic contribution cannot be removed and we obtain the following expression for $a_{1}$ : 


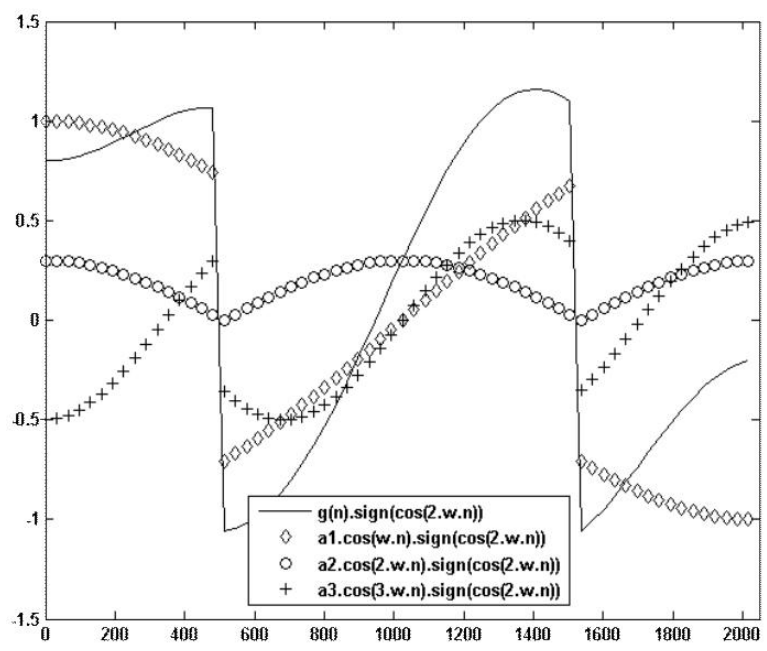

Figure 2. Signals multiplied by the sign of $\cos (2 \omega t)$

$$
a_{1}=\frac{\omega}{4}\left(\sum_{0}^{\frac{N_{s}}{4}} g[n]-\sum_{\frac{N_{s}}{4}}^{\frac{N_{s}}{2}} g[n]\right)-\frac{1}{6} a_{3}
$$

To conclude, we can extract first $a_{2}$ and $a_{3}$ then $a_{1}$ with only three basic cumulative sums from the composite signal $g(t)$, if this signal is composed only of three harmonics. We show in the next section that this technique can be applied to a wideband signal.

2.2.2. General theory. We consider that the signal is limited to the $K_{M A X}$ first harmonics. Based on the technique previously presented, to evaluate the $q^{t h}$ harmonics, we first compute the $G_{q}$ value with the following integral:

$$
G_{q}=\int_{0}^{\frac{N_{s} T_{s}}{2}} g(t) \cdot \operatorname{sign}(\cos (q \omega t)) d t
$$

Thanks to (11), (20) could be rewrote as follows:

$$
\begin{aligned}
G_{q} & =\int_{0}^{\frac{N_{s} T_{s}}{2}}\left(2 \sum_{k=1}^{K_{M A X}}\left(a_{k} \cos (k \omega t)\right)\right) \operatorname{sign}(\cos (q \omega t)) d t \\
& =2 \sum_{k=1}^{K_{M A X}}\left(a_{k} \int_{0}^{\frac{N_{s} \cdot T_{s}}{2}} \cos (k \omega t) \cdot \operatorname{sign}(\cos (q \omega t)) d t\right)
\end{aligned}
$$

Let $C_{q k}$ be the contribution of the $\operatorname{cosine} \cos (k \omega x)$ when the parameter $a_{q}$ is looked for:

$$
C_{q k}=\int_{0}^{\frac{N_{s} T_{s}}{2}} \cos (k \omega t) \cdot \operatorname{sign}(\cos (q \omega t)) d t
$$

then

$$
G_{q}=2 \sum_{k=1}^{K_{M A X}}\left(a_{k} C_{q k}\right)
$$

$G_{q}$ represents the sum of all cosines contribution when the parameter $a_{q}$ is looked for. $A$ matrix product is obtained:

$$
G=\left[\begin{array}{l}
G_{1} \\
\vdots \\
G_{k} \\
\vdots \\
G_{K_{M A X}}
\end{array}\right]=2 C A
$$

with :

$$
C=\left[\begin{array}{ccccc}
C_{11} & \ldots & C_{1 k} & \ldots & C_{1 K_{M A X}} \\
\vdots & & \vdots & & \vdots \\
C_{q 1} & \ldots & C_{q k} & \ldots & C_{q K_{M A X}} \\
\vdots & & \vdots & & \vdots \\
C_{K_{M A X} 1} & \ldots & C_{K_{M A X} k} & \ldots & C_{K_{M A X} K_{M A X}}
\end{array}\right]
$$

and

$$
A=\left[\begin{array}{l}
a_{1} \\
\vdots \\
a_{k} \\
\vdots \\
a_{K_{M A X}}
\end{array}\right]
$$

It is obvious that the function $\operatorname{sign}(\cos (q \omega t))$ only takes 2 values $(1$ or -1$)$, and that these values are constant on the interval described by two consecutives zeros of the function $\cos (q \omega t)$. Thus $C_{q k}$ is evaluated with a simple sum taking into account the zeros of $\cos (q \omega t)$ in the range $\left[0, \frac{N_{s} T_{s}}{2}\right]$. Let $\left(z_{i}\right)_{i \in[1 . . q]}$ be those zeros, and let us add $z_{0}=0, z_{q+1}=\frac{N_{s} . T_{s}}{2}$, the extreme values of the interval, (18) are then expressed by:

$$
C_{q k}=\sum_{i=0}^{q}(-1)^{i} \int_{z_{i}}^{z_{i+1}} \cos (k \omega t) d t
$$

It can be demonstrated that :

- if $k=q$ then

$$
C_{q q}=\frac{2}{\omega}
$$

- if $q$ is odd $(q=2 p+1)$ and if $k$ is odd $(k=2 t+1)$ and if $\frac{k}{q}$ is an odd integer

$$
C_{q k}=(-1)^{p+t} \cdot \frac{2 q}{k \omega}
$$


- if $q$ is even $(q=2 p)$ and if $k$ is even $(k=2 t)$ and if $\frac{k}{q}$ is an odd integer $(k / q=2 m+1)$

$$
C_{q k}=(-1)^{p+t+m} \cdot \frac{2 q}{k \omega}
$$

- else

$$
C_{q k}=0
$$

As matrix $C$ is fully determinate by computation, and as vector $G$ is calculated from data in a very simple way, the estimation of parameters $a_{k}$ is straightforward. Matrix $C$ has to be inverted in order to perform the estimation. As the terms $C_{q k}$ are null for all $k<q$, matrix $C$ is an upper triangular one. Its determinant is thus equal to the product of the diagonals terms, in this case, it is $\left(\frac{2}{\omega}\right)^{K_{M A X}}$. $C$ can then be inverted using the Gauss-Jordan method. The final solution is given by the following equations:

$$
a_{K_{M A X}}=\frac{\omega G_{K_{M A X}}}{4}
$$

for $i \in\left\{1, \ldots, K_{M A X}-1\right\}$

- $i$ even :

$$
a_{i}=\frac{\omega}{4}\left(G_{i}-\sum_{m=1}^{m \leq \frac{1}{2}\left(\frac{K_{M A X}}{i}-1\right)} \frac{2(-1)^{(m+1) i+m}}{(2 m+1) \omega} a_{(2 m+1) i}\right.
$$

- $i$ odd :

$$
a_{i}=\frac{\omega}{4}\left(G_{i}-\sum_{m=1}^{m \leq \frac{1}{2}\left(\frac{K_{M A X}}{i}-1\right)} \frac{2(-1)^{(m+1) i-1}}{(2 m+1) \omega} a_{(2 m+1) i}\right)_{(32)}
$$

Following the same methodology and using $H_{q}=$ $\int_{0}^{\frac{N_{s} \cdot T_{s}}{2}} h(t) \cdot \operatorname{sign}(\sin (q \omega t)) d t$, the estimation of parameters $b_{k}$ is given by the following equations:

$$
b_{K_{M A X}}=\frac{\omega \cdot H_{K_{M A X}}}{4}
$$

for $i \in\left\{1, \ldots, K_{M A X}-1\right\}$

$$
b_{i}=\left(\frac{\omega}{4} H_{i}-\sum_{m=1}^{m \leq \frac{1}{2}\left(\frac{K_{M A X}}{i}-1\right)} \frac{1}{2(2 m+1)} b_{(2 m+1) i}\right)
$$

We can notice that the estimation of $b_{k}$ does not require to distinguish the odd and even cases. This small example demonstrates that with simple processing on the signal to be analysed, it is possible to extract every set of coefficients $a_{k}$ et $b_{k}$ with only cumulative sums and a few multiplications.

\section{Validation}

The previous algorithm was implemented in a MATLAB script and applied to a $12 \mathrm{bits}$ AD converter model. The input signal was a full-scale coherent signal of frequency set around $15 \mathrm{MHz}$, and sampled at $80 \mathrm{MHz}$. Figure 3 shows the result of a FFT operation computed over a sample set of 4096 points.

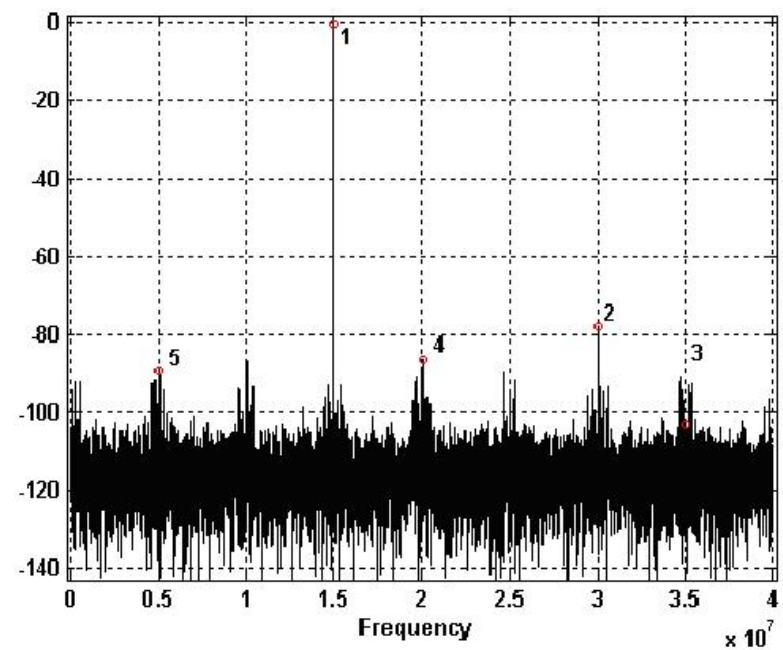

\section{Figure 3. Power spectrum of a12bits AD con- verter}

Figure 4 shows the comparison of the $K_{M A X}=5$ first harmonic estimation for FFT operations computed over sample sets of 4096 points long, and our method, computed over 4096 points. Our method seems to be relevant for the estimation of the five harmonics, even for the lowest ones. The comparison with the FFT operation computed over 4096 points reveals reasonable errors for this real spectrum. Simulations made with an ideal spectrum, where only the five first harmonic get out of the noise floor, give almost perfect results (max mean error $0.5 \%$ and max standard deviation of $6 \%$ on the lasts harmonic). Table 1 shows statistical measurements made over 200 runs for a real spectrum.

Mean and standard deviation operations are computed over the error between the FFT algorithm and our method. All statistical measurements were made on the real values, not the $\mathrm{dBc}$ ones. Our estimation, based on a real spectrum reveals some offset errors, which do not appear in the ideal case. However, even if those errors seem to be important, they represent only a maximum deviation of $3 \mathrm{~dB}$.

The number of multipliers used in this new method is nearly null as they are only used for scaling operations. The number of actual sums required to perform an estimation of the 5 first harmonic over a 4096 points long sample set is around 40,000. When comparing this to the 53,256 


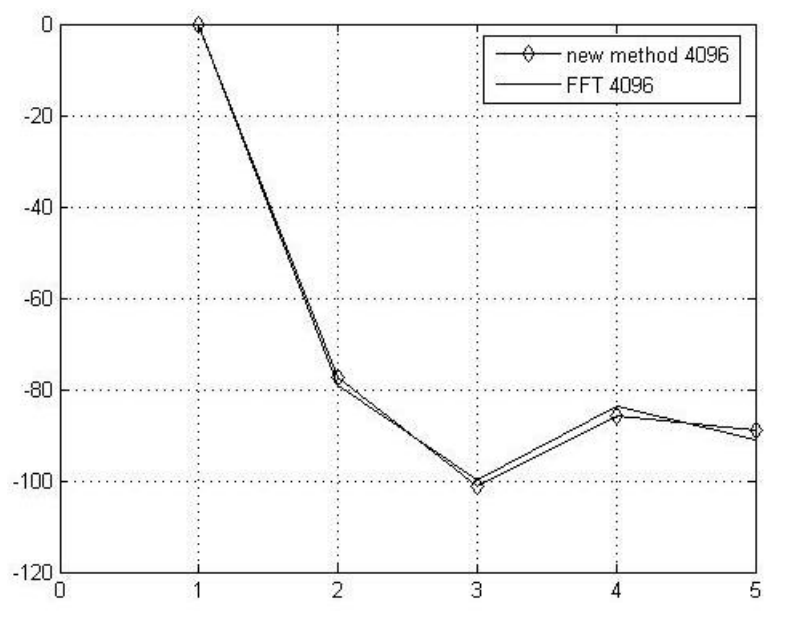

Figure 4. Comparisons between FFT and our method

Table 1. Comparisons and errors

\begin{tabular}{||c||c|c|c|c|c||}
\hline Harm. & $H_{1}$ & $H_{2}$ & $H_{3}$ & $H_{4}$ & $H_{5}$ \\
\hline FFT & 0 & $-77,32$ & $-101,13$ & $-85,95$ & $-89,06$ \\
\hline Our method & 0 & $-79,04$ & $-99,98$ & $-83,70$ & $-91,10$ \\
\hline $\bar{E}(\%)$ & $-0,0020$ & 17,97 & $-12,32$ & $-29,59$ & 21,08 \\
\hline$\sigma_{E}(\%)$ & 0,00022 & 1,320 & 28,282 & 3,88 & 5,329 \\
\hline
\end{tabular}

products and the 126, 296 adds needed for a 4096 Radix-4 FFT operation.

It is clear that the silicon area required to implement the harmonic estimation is directly linked with the number and the nature of the needed operators. For The same accuracy as the classical FFT test technique, because our method needs very fewer operations and only real additions, we can expect obtain a good result for the first implementation of our method with a small silicon area compatible with BIST constraints.

\section{Conclusion}

In this paper, a new power estimation algorithm of the first harmonics that respects the constraints of an embedded process has been exposed. The method, based on discrete Fourier series expansion model, and piecewise signal decomposition consists in a very simple signal processing. First studies are encouraging to follow in this way. We are currently working on the implementation of the algorithm in a FPGA solution to quantify more precisely, the hardware resource needed. Furthermore, the validation of the algorithm was leaded with a single sinusoid in input, it will be interesting to check the behavior of the algorithm for a larger variety of signals (for instance, two-tone signals).
Next step will be to detail the BIST circuitry to perform the operation.

\section{Acknowledgements}

This work has been carried out under frame of the European MEDEA+ Project: "Nanotest".

\section{References}

[1] J.-M. Janik, V. Kerzerho, and V. Fresnaud. A spectral approach to estimate the inl of a/d converter. IEEE Trans. Instrum. Meas (to be published).

[2] F. Attivissimo, N. Giaquinto, and I. Kale. Inl reconstruction of $\mathrm{a} / \mathrm{d}$ converters via parametric spectral estimation. IEEE Trans. on Instrumentation and Measurement, Vol 53:pp940946, August 2004.

[3] L. Marple. A new autoregressive spectrum analysis algorithm. IEEE Trans. on Acoustics, Speech, and Signal Processing, Vol 28:pp441-454, August 1980.

[4] J. Kulubi and L. van Biesen. A unified framework for knowledge based spectrum analysis. IEEE Trans. on Instrumentation and Measurement, Vol 39:pp827-834, December 1990.

[5] S. Marple. Digital Spectral Analysis. Englewood Cliffs, 1987.

[6] C. Rebai, D. Dallet, and P. Marchegay. Noncoherent spectral analysis of adc using filter bank. IEEE Transactions on Instrumentation and Measurement, Vol 53:pp652-660, June 2004.

[7] D. Dallet, C. Rebai, V. Boun, D. Elfersi, A. Merino, and P. Marchegay. Adaptive filtering for adc spectral analysis. International Workshop on ADC Modeling and Testing (IWADC), 2003.

[8] D. Vazquez, G. Leger, G. Huertas, A. Rueda, and J. Huertas. Sine-wave signals characterization using first order $\sigma \delta$ modulators: Application to mixed-signal bist. IEEE International Mixed-Signal Workshop, 2003.

[9] D. Vazquez, G. Leger, G. Huertas, A. Rueda, and J. Huertas. Benchmarking of fft algorithms. Proc. Design, Automation and Test in Europe Conference and Exhibition, pp298-303, 2004.

[10] J. Proakis and D. Manolakis. Digital Signal Processing: Principles, Algorithms and Applications. Englewood Cliffs, 1996.

[11] IEEE Standard 1241-2000. IEEE standard for terminology and test methods for analog- to- digital converters, June 2001.

[12] J. Cooley and J. Tukey. An algorithm for the machine calculation of fourier series. Math. Comput., Vol 19:pp297-301, 1965.

[13] Y. Chan and R. Langford. Spectral estimation via the highorder yule-walker equations. IEEE Transactions on Signal Processing, Vol 30:pp689-698, October 1982.

[14] M. Balducci, A. Ganapathiraju, J. Hamaker, J. Picone, A. Choudary, and A. Skjellum. Benchmarking of fft algorithms. IEEE Southeastcon '97. Engineering new New Century, pages pp328-330, April 1997.

[15] M. Mahoney. DSP-Based Testing of Analog and MixedSignal Circuits. Wiley-IEEE Computer Society Press, 1987. 